EOMmUn: Communication et organisation

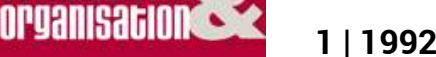

Communication et sentiment d'appartenance

\title{
Des cercles en scène chez Sony
}

Gino Gramaccia

\section{OpenEdition}

Journals

Édition électronique

URL : http://journals.openedition.org/communicationorganisation/1571

DOI : 10.4000/communicationorganisation. 1571

ISSN : 1775-3546

Éditeur

Presses universitaires de Bordeaux

Édition imprimée

Date de publication : 1 mai 1992

ISSN : 1168-5549

Référence électronique

Gino Gramaccia, «Des cercles en scène chez Sony », Communication et organisation [En ligne], 1 | 1992, mis en ligne le 26 mars 2012, consulté le 19 avril 2019. URL : http://journals.openedition.org/ communicationorganisation/1571; DOI : 10.4000/communicationorganisation.1571

Ce document a été généré automatiquement le 19 avril 2019

(c) Presses universitaires de Bordeaux 


\title{
Des cercles en scène chez Sony
}

\author{
Gino Gramaccia
}

1 Biarritz, Palais des Festivals, un samedi en décembre : Sony présente, en 1991, la $6^{\mathrm{e}}$ Journée de la Qualité. Point d'orgue des activités des cercles de qualité des sites de Bayonne et de Dax, cette journée est conçue comme un "spectacle didactique " (la formule, on va le voir, est assez juste) dont l'objectif le plus novateur, et assurément le plus riche d'effets de sens, consiste à mettre en scène la parole ouvrière. Cette pratique s'inscrit dans la logique méthodique d'une Charte et d'une expérience de sept années très concluante des cercles de qualité.

2 Chez Sony en Aquitaine ${ }^{1}$ les premiers cercles ont été lancés en 1983. Le Comité de Coordination des Cercles de Qualité (C.C.C.Q.) voit le jour en 1985 avec pour mission de coordonner le travail des cercles sur les deux sites - Bayonne et Dax - et d'organiser les actions de formation et de valorisation. La Charte des Cercles de qualité est alors édictée. La première Journée date de décembre 1986. À la fin de l'année 1987, douze cercles fonctionnent dans les principaux ateliers des usines et, en 1991, on compte au total cinquante-huit cercles créés en sept ans impliquant plus de 350 personnes.

3 La pratique des cercles est devenue, dans ce contexte très précis, et comme il se doit, une technologie manageriale bien rôdée. C'est en tout cas l'avis de Christian Darmaillacq, Gérard Dulin et Jean-Paul Saint-Martin, cadres des usines de Dax et de Bayonne ${ }^{2}$, qui ont largement contribué, au sein des Comités de coordination, au développement de ce qui peut être considéré à juste titre comme un moyen efficace de communication interne. Les effets d'apprentissage générateurs d'une dynamique de groupe, d'un climat social favorable à la création de liens informels, d'une parole collective qui abolit cloisonnements et distances, y sont sans doute pour quelque chose.

Un examen plus précis des conditions qui ont permis d'assurer la pérennité de ces cercles dépasserait les limites de cette petite étude. On indiquera simplement que les cercles sont hétérogènes, rassemblant, autour d'une majorité d'employés d'un secteur spécifique (production, par exemple) des intervenants de services différents (administration, qualité, équipement...). L'apport des savoirs utiles au fonctionnement des cercles est d'une flexibilité étonnante: on a besoin d'un expert financier? On fait appel au comptable de la société. Besoin d'un savoir-faire spécifique? Un opérateur du secteur 
voisin se rend disponible. Bien entendu, l'activité pratique des cercles se nourrit en permanence d'éléments de formation à la qualité proposés par les animateurs. Irréprochable dans sa démarche, le Comité de coordination veille à l'application des solutions proposées, mais avant tout, à leur faisabilité. L'orthodoxie de la procédure est donc intégralement préservée. Une parole transversale circule, qui élabore sa cohérence propre, décloisonne l'approche des problèmes, génère des rapports sociaux intégrant la dimension normative de modèles de gestion (diagrammes P.E.R.T., Causes/effet, Pareto... ). Ce qui, au fond, devient significatif, c'est la volonté de pérenniser la pratique des cercles par ailleurs en perte de vitesse dans des sociétés qui ont largement contribué à leur promotion (Ford, IBM).

5 Revenons à la Journée de présentation des cercles à laquelle j'ai eu le privilège d'assister en qualité d'observateur critique ${ }^{3}$. Je ne me livrerai pas à une quelconque analyse comparative ou constat détaillé de progrès entre 1990 et 1991, ce qui serait fastidieux, mais au descriptif rapide de la Journée du 7 décembre 1991.

Dans le décor rénové du Palais des Festivals - qui a gardé en façade le charme de la gare ancienne - rien n'est laissé au hasard. Le matin, les cercles répètent dans une ambiance de concert rock : c'est la «balance», dans le jargon technique des opérateurs de concert. Projecteurs de poursuite, caméras (Sony oblige!), sonorisation sophistiquée, système de projection multimédia, régie son et image, tout est pensé, tout est construit pour donner au spectacle qui va suivre dans l'après-midi cette force mythique des effets d'amplification et de filtrage phonique et lumineux.

7 Le spectacle ne déçoit pas. Dans la salle, les huit cents invités (les personnels des usines de Dax, de Bayonne et Rovereto (Italie)) auront la spontanéité et l'enthousiasme adolescent du public de Patrick Bruel. Sur scène, le rythme cadencé, contrasté, paradoxal, de tous les discours. Celui, inaugural, de M. Kuga, responsable marketing au niveau mondial (pour les produits magnétiques): il s'exprime en Anglais mais la traduction, assurée par les animateurs, intervient en séquences alternées; l'allocution de la hiérarchie française (M. Galiana-Mingot ${ }^{4}$ et de M. Ehret ${ }^{5}$ ), aux accents toniques de l'épopée mais teintés de réalisme ; le discours des opérateurs présentant, en une solennelle attitude, les travaux de leurs cercles ; l'oraison efficace de Jean-Paul Loth, ex-entraîneur de l'équipe de France de Tennis, développant la métaphore sportive de l'effort récompensé.

8 Mais la part la plus belle est donnée, de la manière la plus légitime, aux acteurs des cercles de qualité. Le discours est construit, appliqué, ciselé comme une phrase de Flaubert, mais aussi agité par toutes les peurs le micro VHF passe de mains en mains, à la mode olympique. Les commentaires de diagrammes sont doctes comme des soutenances, et la voix, amplifiée, mixée, soudain synthétique, soudain naturelle, a la force émotive d'une confession.

9 Un autre langage ajoute à la complexité de ces messages multiples, conjugués, contribuant à tisser sans doute ce qu'il est convenu d'appeler la "culture Sony ", du moins telle qu'elle est vécue et partagée chez Sony Aquitaine: celui des petits sujets vidéo, filmés par les cercles eux-mêmes, avec des moyens spécifiques et précédant, en exergue, toutes les prestations orales. Des chefs-d'œuvre d'humour ramenant, involontairement sans doute, le spectateur à la dimension d'un autre code : celui qui permet de mieux comprendre la genèse de ce « spectacle didactique » en révélant, au plus près, les savoir-faire des opérateurs, leur culture de groupe, leur complicité et leur lucidité. L'illustration musicale de ces films? Surtout des pasos dobles. Alors la salle se met à chanter. Dans cette cacophonie de grand style, aucune hypothèse n'est 
vraisemblable. Le repas convivial dans la soirée, joyeux, musical, dansant, explosif parfois, finit d'égarer mes prétentions à l'analyse. Le lundi suivant, trois nouveaux cercles se portent volontaires à l'usine de Dax.

\section{NOTES}

1. Sony en Aquitaine regroupe Les sites de Bayonne (usine de cassettes audio et usine de composants), qui comptent au total 600 personnes, et le site de Dax/Pontonx (usine de cassettes vidéo et usine d'enduction), employant 350 salariés.

2. Christian Darmaillacq est responsable du service Logistique, Gérard Dulin (Dax) et Jean-Paul Saint Martin (Bayonne), responsables de Production.

3. Dana un souci de perfection d'autant plus louable qu'il visait à briser la boucle si rassurante de l'auto-congratulation, les promoteurs de la journée ont pris l'initiative de réunir un groupe d'observateurs extérieurs (composé pour l'essentiel de consultants et de formateurs). Cet «œil extérieur" - c'est son nom - avait pour mission de critiquer, à des fins d'amélioration, l'ensemble des prestations.

4. Egalement directeur de la Division Produits magnétiques Europe.

5. Directeur Général des activités composantes et supports d'enregistrements.

\section{RÉSUMÉS}

Les cerles de qualité chez Sony Aquitaine, sont mis en scène : voilà le temps fort de la Journée Qualité, "Convention-Show » authentique, qui réunit, en des salles prestigieuses, tous les personnels des sites de Dax et de Bayonne. Les cercles, technologie managériale très au point chez Sony, passent très bien la rampe.

\section{AUTEUR}

\section{GINO GRAMACCIA}

Gino Gramacia est maître de conférences à l'Institut Universitaire de Technologie « A » de Bordeaux. Il enseigne la Communication et la Gestion de Projet dans le Département de Génie Electrique et d'Informatique Industrielle. Il travaille actuellement sur le projet de communication externe des PME/PMI en direction des partenaires universitaires (dernière publication à paraître : «Vivre les relations École/Entreprise : une expérience du produire » ETVDES été 92). 